\title{
CURVATURE INHERITANCE SYMMETRY IN RIEMANNIAN SPACES WITH APPLICATIONS TO STRING CLOUD AND STRING FLUIDS
}

\author{
İ. Yılmaz ${ }^{1}$, I. $\operatorname{Yavuz}^{2}$, H. Baysal ${ }^{3}$, I. $\operatorname{Tarhan}^{1}$ and U. Camci ${ }^{3}$ \\ ${ }^{1}$ Department of Physics, Faculty of Arts and Sciences, Çanakkale \\ Onsekiz Mart University, 17100 Çanakkale, Turkey. \\ e-mail: iyilmaz@hotmail.com and tarhan_ism@hotmail.com \\ ${ }^{2}$ Department of Computer Engineering, Faculty of Engineering \\ and Architecture, Çanakkale Onsekiz Mart University, 17100 \\ Çanakkale, e-mail: yavuz@hotmail.com \\ ${ }^{3}$ Department of Mathematics, Faculty of Arts and Sciences, \\ Çanakkale Onsekiz Mart University, 17100 Çanakkale, Turkey. \\ e-mail: hbaysal@hotmail.com and ucamci@hotmail.com
}

\begin{abstract}
We study, in this paper, curvature inheritance symmetry (CI), $£_{\xi} R_{b c d}^{a}=2 \alpha R_{b c d}^{a}$, where $\alpha$ is a scalar function, for string cloud and string fluid in the context of general relativity. Also, we have obtained some result when a proper CI (i.e., $\alpha \neq 0$ ) is also a conformal Killing vector.
\end{abstract}

Key Words: Cosmic Strings, Inheritance symmetry, Riemann Space, Cosmology 


\section{Introduction}

The question of symmetry inheritance is concerned with determining when the symmetries of geometry ( defined through the existence of symmetry vectors) are inherited by the source terms or individual physical components of the energy-stress tensor (related to the geometry via Einstein field equation).

The most useful inheritance symmetry is the symmetry under the conformal motions (Conf $\mathrm{M}$ ). A $V_{n}$ admits a Conf $\mathrm{M}$ generated by a conformal Killing vector $(\mathrm{CKV}) \xi$ if

$$
£_{\xi} g_{a b}=2 \psi g_{a b}, \psi=\psi\left(x^{a}\right) .
$$

It follows from Yano ${ }^{1}$ that every Conf M must satisfy

$$
\begin{gathered}
£_{\xi} \Gamma_{b c}^{a}=\delta_{b}^{a} \psi_{; c}+\delta_{c}^{a} \psi_{; b}-g_{b c} g^{a d} \psi_{; d} \\
£_{\xi} R_{a b}=-g_{a b} \square \psi-(n-2) \psi_{; a b} \\
£_{\xi} R=-2(n-1) \square \psi-2 \psi R \\
£_{\xi} C_{b c d}^{a}=0
\end{gathered}
$$

where $£_{\xi}$ signifies the Lie derivative along $\xi^{a}$ and $\psi\left(x^{a}\right)$ is the conformal factor and $\square$ is the Laplacian operator defined by $\square \psi=g^{a b} \psi_{; a b}$. In particular, $\xi$ is special conformal Killing vector (SCKV) if $\psi_{; a b}=0$ and $\psi_{, a} \neq 0$. Other subcases are homothetic vector $(\mathrm{HV})$ if $\psi_{, a}=0$ and Killing vector (KV) if $\psi=0$. Here $(;)$ and $($,$) denote the covariant and ordinary derivatives,$ respectively.

The study of inheritance symmetries with CKV's and SCKV in fluid space-times (perfect, anisotropic, viscous and heat-conducting) has recently attracted some interest. Herrera et al. ${ }^{2}$ have studied CKV's, with particular reference to perfect and anisotropic fluids; Mason and Tsamparlis ${ }^{3}$ have investigated spacelike CKV's; Maartens et al. ${ }^{4}$ have made a study of CKV's in anisotropic fluids, in which they are particularly concerned with special conformal Killing vector (SCKV); Coley and Tupper ${ }^{5}$ have discussed spacetimes admitting SCKV and symmetry inheritance. Yavuz and Yand special conformal Killing vectors in string cosmology. Carot et al. ${ }^{7}$ have discussed space-times with conformal Killing vectors. Also, Duggal ${ }^{8,9}$ have discussed curvature inheritance symmetry in Riemannian spaces with applications to fluid space-times. Recently, the Einstein field equations for inhomogeneous 
cylindrically symmetric space-times filled cosmic strings have been discussed by Kılınc and Yavuz ${ }^{10}$. Some string dust models for Bianchi Type I spacetime have been studied in detail by Yavuz and Tarhan ${ }^{11}$. The Einstein's field equations for a cloud of string with heat flux in Bianchi Type III space-time solved by Yavuz and Yllmaz ${ }^{12}$. Bianchi Type I universes representing different forms of material distributions have been studied by Bali and Jain ${ }^{13}$, Bali and Tyagi ${ }^{14}$, Bali ${ }^{15}$, Singh and Srivastava ${ }^{16}$, Bali and Deepak ${ }^{17}$, Kllınc ${ }^{18}$ (see also references therein).

In this paper, we will examine curvature inheritance symmetry in the space-times with string source (string cloud and string fluid). Energy momentum tensor for a cloud of strings can be written as

$$
T_{a b}=\rho u_{a} u_{b}-\lambda x_{a} x_{b}
$$

where $\rho$ is the rest energy for cloud of strings with particles attached to them and $\lambda$ is string tensor density and are related by

$$
\rho=\rho_{p}+\lambda \text {. }
$$

Here $\rho_{p}$ is particle energy density. The unit timelike vector $u^{a}$ describes the cloud four-velocity and the unit spacelike vector $x^{a}$ represents a direction of anisotropy, i.e., the string's directions ${ }^{19}$. We have

$$
u^{a} u_{a}=-x^{a} x_{a}=-1 \text { and } u^{a} x_{a}=0 .
$$

The energy-momentum tensor for a fluid of strings ${ }^{20,21}$ is

$$
T_{a b}=\left(q+\rho_{s}\right)\left(u_{a} u_{b}-x_{a} x_{b}\right)+q g_{a b}
$$

Also, note that

$$
u^{a} u_{a}=-x^{a} x_{a}=-1 \text { and } u^{a} x_{a}=0
$$

where $\rho_{s}$ is string density and $q$ is "string tension" and also "pressure".

The paper may be outlined as follows. In Section II curvature inheritance equations in the cloud of string and in Section III equations state of string cloud are obtained. In Section IV curvature inheritance equations in the fluids of string are derived. In Section $\mathrm{V}$ the results are discussed. 


\section{Curvature Inheritance Symmetry In The Cloud of Strings}

Consider a Riemannian space $V_{n}$ of arbitrary signature. We define symmetry called "curvature inheritance" (CI) on $V_{n}$ by an infinitesimal transformation $\bar{x}^{a}=x^{a}+\xi^{a}(x) \delta(t)$, for which

$$
£_{\xi} R_{b c d}^{a}=2 \alpha R_{b c d}^{a}
$$

where $\alpha=\alpha(x)$ is a scalar function, $\delta(t)$ is a positive infinitesimal and $R_{b c d}^{a}$ is the Riemannian curvature tensor defined by

$$
R_{b c d}^{a}=\Gamma_{b d, c}^{a}-\Gamma_{b c, d}^{a}+\Gamma_{b d}^{e} \Gamma_{e c}^{a}-\Gamma_{b c}^{e} \Gamma_{e d}^{a}
$$

Here $\Gamma_{b c}^{a}$ are the Christoffel symbols of the second kind. A subcase of CI is the well-known symmetry "curvature collineation" (CC) when $\alpha=0$. In

the sequel, we say that CI is proper if $\alpha \neq 0$. If a $V_{n}$ admits a CI, then the following identities hold $\left(£_{\xi} g_{a b} \equiv h_{a b}\right)$ :

$$
£_{\xi} R_{a b}=2 \alpha R_{a b},
$$

i.e., $\xi$ defines Ricci inheritance symmetry,

$$
\begin{gathered}
£_{\xi} R_{b}^{a}=2 \alpha R_{b}^{a}-R_{b}^{c} h_{c}^{a}, \\
£_{\xi} R=2 \alpha R-R^{\prime}, \\
£_{\xi} W_{b c d}^{a}=2 \alpha W_{b c d}^{a}, \\
£_{\xi} C_{b c d}^{a}=2 \alpha C_{b c d}^{a}+D_{b c d}^{a},
\end{gathered}
$$

where $R$ is the scalar curvature, $R^{\prime}=R_{a b} h^{a b}$ and

$$
\begin{aligned}
W_{b c d}^{a}= & R_{b c d}^{a}-\frac{1}{n-1}\left[\delta_{d}^{a} R_{b c}-\delta_{c}^{a} R_{b d}\right]: \text { Weyl projective tensor } \\
C_{b c d}^{a}= & R_{b c d}^{a}+\frac{1}{n-2}\left[\delta_{c}^{a} R_{b d}-\delta_{d}^{a} R_{b c}+g_{b d} R_{c}^{a}-g_{b c} R_{d}^{a}\right] \\
& \left.+\frac{R}{(n-1)(n-2)}\left[\delta_{d}^{a} g_{b c}-\delta_{c}^{a} g_{b d}\right]: \text { Conformal curvature tens } \phi \mathbb{1}, 9\right)
\end{aligned}
$$




$$
\begin{aligned}
D_{b c d}^{a}= & \frac{1}{n-2}\left[h_{b d} R_{c}^{a}-h_{b c} R_{d}^{a}+g_{b c} R_{d}^{e} h_{e}^{a}-g_{b d} R_{c}^{e} h_{e}^{a}\right] \\
& +\frac{1}{(n-1)(n-2)}\left[\delta_{d}^{a}\left(R h_{b c}-R^{\prime} g_{b c}\right)-\delta_{c}^{a}\left(R h_{b d}-R^{\prime} g_{b d}\right)\right]
\end{aligned}
$$

A CI is also a $\mathrm{CKV}^{7,8}$ if

$$
\begin{gathered}
\psi_{; a b}=\frac{\alpha}{n-2}\left[\frac{R}{n-1} g_{a b}-2 R_{a b}\right] \\
\square \psi+\frac{\alpha R}{n-1}=0 \\
\alpha=\psi+\xi^{a} \partial_{a}(\log \sqrt{R})
\end{gathered}
$$

The proof of Eq. (21) follows by comparing (13), (15) with (3). Also, the proof of Eq. (22) follows from (4) and (21). If $X^{a}$ is any unit vector (timelike or spacelike) and $\xi^{a}$ is a CKV satisfying (1), then

$$
\begin{gathered}
£_{\xi} X^{a}=-\psi X^{a}+Y^{a}, \\
£_{\xi} X_{a}=\psi X_{a}+Y_{a},
\end{gathered}
$$

where $Y^{a}$ is some vector orthogonal to $X^{a}$, i.e., $X^{a} Y_{a}=0$, (see Ref. 3).

Applying the results (24) and (25) to the timelike unit four-velocity vector $u^{a}$ and to spacelike unit vector $x^{a}$ of the string cloud and string fluid, we have

$$
\begin{gathered}
£_{\xi} u^{a}=-\psi u^{a}+v^{a}, \\
£_{\xi} u_{a}=\psi u_{a}+v_{a},
\end{gathered}
$$

where $u_{a} v^{a}=0$ and

$$
\begin{gathered}
£_{\xi} x^{a}=-\psi x^{a}+n^{a}, \\
£_{\xi} x_{a}=\psi x_{a}+n_{a},
\end{gathered}
$$

where $x_{a} n^{a}=0$. Since $x_{a} u^{a}=0$ [see eq.(8)] we have 


$$
x_{a} £_{\xi} u^{a}+u^{a} £_{\xi} x_{a}=0 .
$$

Substituting Eqs. (26) and (29) into (30), we get

$$
v_{a} x^{a}+n_{a} u^{a}=0 .
$$

If $\xi^{a}$ is a CKV satisfying (1), then

$$
\begin{gathered}
£_{\xi} R_{a b}=-2 \psi_{; a b}-g_{a b} \square \psi, \\
£_{\xi} R=-2 \psi R-6 \square \psi, \\
£_{\xi} G_{a b}=2 g_{a b} \square \psi-2 \psi_{; a b},
\end{gathered}
$$

where $\square \psi \equiv g^{a b} \psi_{; a b}, R_{a b}$ is Ricci tensor, $G_{a b}$ is Einstein tensor and $R=$ $g^{a b} R_{a b}$ is Ricci scalar. Via Einstein's field equations

$$
G_{a b} \equiv R_{a b}-\frac{1}{2} R g_{a b}=T_{a b}
$$

we find for $T_{a b}$

$$
£_{\xi} T_{a b}=2 g_{a b} \square \psi-2 \psi_{; a b}
$$

We take $T_{a b}$ to be of the form (6). With the aid of (27) for $£_{\xi} u_{a}$ and (29) for $£_{\xi} x_{a}$ a direct calculation yields

$$
£_{\xi} T_{a b}=\left[£_{\xi} \rho+2 \psi \rho\right] u_{a} u_{b}-\left[£_{\xi} \lambda+2 \psi \lambda\right] x_{a} x_{b}+2 \rho u_{(a} v_{b)}-2 \lambda x_{(a} n_{b)},
$$

which, when substituted into (36), gives

$$
\begin{aligned}
2 \square \psi\left(h_{a b}-u_{a} u_{b}+x_{a} x_{b}\right)-2 \psi_{; a b}= & {\left[£_{\xi} \rho+2 \psi \rho\right] u_{a} u_{b}-\left[£_{\xi} \lambda+2 \psi \lambda\right] x_{a} x_{b} } \\
& +2 \rho u_{(a} v_{b)}-2 \lambda x_{(a} n_{b)}
\end{aligned}
$$

where $h_{a b}$ is projection tensor that projects in the directions that are perpendicular to both $x^{a}$ and $u^{a}$,

$$
h_{a b}=g_{a b}+u_{a} u_{b}-x_{a} x_{b} .
$$


Some properties of this tensor are

$$
\begin{gathered}
h^{a b} u_{b}=h^{a b} x_{b}=0 \\
h_{c}^{a} h_{b}^{c}=h_{b}^{a}, h_{a b}=h_{b a}
\end{gathered}
$$

By contracting (38) with the tensors $u^{a} u^{b}, x^{a} x^{b}, u^{a} x^{b}, u^{a} h^{b c}$ and $x^{a} h^{b c}$ the following equations for the string cloud are derived:

$$
\begin{gathered}
£_{\xi} \rho+2 \psi \rho=-2\left(\square \psi+\psi_{; a b} u^{a} u^{b}\right) \\
£_{\xi} \lambda+2 \psi \lambda=2\left(\square \psi-\psi_{; a b} x^{a} x^{b}\right) \\
(\rho-\lambda) x^{b} v_{b}=\rho_{p} x^{b} v_{b}=2 \psi_{; a b} u^{a} x^{b} \\
\rho h^{b c} v_{b}=2 \psi_{; a b} u^{a} h^{b c} \\
\lambda h^{b c} n_{b}=2 \psi_{; a b} x^{a} h^{b c}
\end{gathered}
$$

Equations (42)-(46) are valid for any CKV $\xi^{a}$. For the string cloud, using the Einstein equations (35), we obtain

$$
R_{a b} u^{a} u^{b}=\rho-R / 2, R_{a b} x^{a} x^{b}=R / 2-\lambda .
$$

It, therefore, follows from (21), (22) and (47) that

$$
\begin{gathered}
\psi_{; a b} u^{a} u^{b}=\alpha(R / 3-\rho), \psi_{; a b} x^{a} x^{b}=\alpha(\lambda-R / 3), \\
\psi_{; a b} u^{a} x^{b}=0, \psi_{; a b} u^{a} h^{b c}=0, \psi_{; a b} u^{a} h^{b c}=0 .
\end{gathered}
$$

Using (48) and $\square \psi=-\frac{1}{3} \alpha R$ [see Eq.(22)], from Eqs. (42) and (43) we have

$$
\begin{gathered}
£_{\xi} \rho=2 \rho(\alpha-\psi), \\
£_{\xi} \lambda=-2 \lambda(\alpha+\psi) .
\end{gathered}
$$

Now, due to (49), Eqs. (44), (45) and (46) reduce to 


$$
\begin{gathered}
\rho_{p} x^{b} v_{b}=0, \\
\rho h^{a b} v_{b}=0, \\
\lambda h^{a b} n_{b}=0 .
\end{gathered}
$$

If we consider (52) and (53) and assume that

$$
\rho_{p} \neq 0 \text { and } \rho \neq 0
$$

then from (52) and (53) we have

$$
x^{b} v_{b}=0, h^{a b} v_{b}=0 .
$$

Since $u^{b} v_{b}=0$ we conclude therefore that $v^{b} \equiv 0$. Equations (26) and (27) reduce to

$$
\begin{gathered}
£_{\xi} u^{a}=-\psi u^{a}, \\
£_{\xi} u_{a}=\psi u_{a} .
\end{gathered}
$$

If we consider (54) and assume that

$$
\lambda \neq 0
$$

Then we have

$$
h^{a b} n_{b}=0 .
$$

We also have $x^{b} n_{b}=0$ and further from (31)

$$
u^{b} n_{b}=-x^{b} v_{b}
$$

But if $\rho_{p} \neq 0$ it follows from (52) that $x^{b} v_{b}=0$ and therefore $u^{b} n_{b}=0$ by (60). Thus since $h^{a b} n_{b}=0, x^{b} n_{b}=0$, and $u^{b} n_{b}=0$ we conclude that $n_{b} \equiv 0$. In this case, (28) and (29) reduce to

$$
£_{\xi} x^{a}=-\psi x^{a}
$$




$$
£_{\xi} x_{a}=-\psi x_{a} .
$$

Also, the derivations of (58) required the reasonable assumptions contained in (55), and (63) required $\lambda \neq 0$ and $\rho_{p} \neq 0$.

\section{Equations of State}

Let us give an example how the inheritance quantities are useful for deriving equations of state in the cloud of strings. We will find these for many special cases, i.e., $\xi$ is parallel to $u^{a}$ and $x^{a}$ and orthogonal to both $x^{a}$ and $u^{a}$. We assume a $\mathrm{CKV} \xi$ is also a CI. If $\xi$ is a CKV satisfying (1), then

$$
\left(R^{a b} \xi_{b}\right)_{; a}=-3 \square \psi
$$

Via Einstein's field equations

$$
R^{a b}=T^{a b}-\frac{1}{2} T g^{a b}
$$

we find

$$
\left[\left(T^{a b}-\frac{1}{2} T g^{a b}\right) \xi_{b}\right] ; a=-3 \square \psi .
$$

Using the Einstein's equations (35), $3 \square \psi=-\alpha R$, equation (66) reduces to

$$
\left[\left(T^{a b}-\frac{1}{2} T g^{a b}\right) \xi_{b}\right]_{; a}=\alpha R
$$

For a cloud of strings with energy-momentum tensor (6) we have

$$
\left(T^{a b}-\frac{1}{2} T g^{a b}\right) \xi_{b}=\frac{1}{2}(\rho-\lambda) u^{a}\left(u^{b} \xi_{b}\right)+\frac{1}{2}(\rho-\lambda) x^{a}\left(x^{b} \xi_{b}\right)+\frac{1}{2}(\rho+\lambda) h^{a b} \xi_{b} .
$$

First, suppose that $\xi^{a}$ is parallel to $x^{a}: \xi^{a}=\xi x^{a}$. Then since $u^{b} x_{b}=0$ and $h^{a b} x_{b}=0,(68)$ reduces to

$$
\left(T^{a b}-\frac{1}{2} T g^{a b}\right) \xi_{b}=\frac{1}{2}(\rho-\lambda) \xi^{a},
$$

which, when substituted into (67), gives 


$$
£_{\xi} \rho-£_{\xi} \lambda+(\rho-\lambda) \xi_{; a}^{a}=0
$$

But from (1),

$$
\xi_{; a}^{a}=4 \psi
$$

and using also (50), (51), and $R=\rho+\lambda$, Eq. (50) becomes

$$
2 \psi \rho=2 \psi \lambda
$$

From Eq. (72) we have

$$
\rho=\lambda \text {. }
$$

Second, suppose that $\xi^{a}$ is parallel to $u^{a}$. Equation (68) reduces to

$$
\left(T^{a b}-\frac{1}{2} T g^{a b}\right) \xi_{b}=-\frac{1}{2}(\rho-\lambda) \xi^{a}
$$

and proceeding as previously we find that

$$
\psi(\rho-\lambda)=-2 \alpha(\rho+\lambda) .
$$

Finally, suppose that $\xi^{a}$ is orthogonal to $x^{a}$ and $u^{a}$. Then $h^{a b} \xi_{b}=\xi^{a}$ and (68) becomes

$$
\left(T^{a b}-\frac{1}{2} T g^{a b}\right) \xi_{b}=\frac{1}{2}(\rho+\lambda) \xi^{a} .
$$

On substituting (76) into (67) and proceeding as before we obtain

$$
\psi(\rho-\lambda)=2 \alpha \lambda
$$

For $\alpha \neq 0$ (i.e., proper CI), Eq. (77) provide physically meaningful equation of state for a give $\alpha$ and $\psi$. To illustrate this point, consider a simple case for which

$$
\alpha=\frac{w}{2} \psi
$$

where $w$ is constant and $w>0$. Eq. (77) provide the following equation of state for $\psi \neq 0$ 


$$
\rho=(1+w) \lambda \text {. }
$$

From Eq. (73) we have state equations $(\rho=\lambda)$ which coincide with the state equation for a cloud of geometric (Nambu) strings ${ }^{19}$. Also, from Eq. (79) we have state equations $(\rho=(1+w) \lambda)$ which coincide with Takabayashi string $^{19}$.

\section{Curvature Inheritance Symmetry In The Fluids of Strings}

In this section we will consider the fluids of strings described by energy-

momentum tensor (9). With the aid of (27) for $£_{\xi} u_{a}$ and (29) for $£_{\xi} x_{a}$ a direct calculation yields

$$
\begin{aligned}
£_{\xi} T_{a b}= & {\left[£_{\xi} \rho_{s}+2 \psi 2 \rho_{s}\right] u_{a} u_{b}-\left[£_{\xi} \rho_{s}+2 \psi \rho_{s}\right] x_{a} x_{b} } \\
& \left.+\left[£_{\xi} q+2 \psi\right]\right] h_{a b}+\left[2 \rho_{s}+2 q\right] u_{(a} v_{b)}-\left[2 \rho_{s}+2 q\right] x_{(a} n_{b)},
\end{aligned}
$$

which, when substituted into (36), gives

$$
\begin{aligned}
2 \square \psi\left(h_{a b}-u_{a} u_{b}+x_{a} x_{b}\right)-2 \psi_{; a b}= & {\left[£_{\xi} \rho_{s}+2 \psi \rho_{s}\right] u_{a} u_{b}-\left[£_{\xi} \rho_{s}\right.} \\
& \left.+2 \psi \rho_{s}\right] x_{a} x_{b}+\left[£_{\xi} q+2 \psi q\right] h_{a b} \\
& \left.+\left[2 \rho_{s}+2 q\right] u_{(a} v_{b}\right) \\
& -\left[2 \rho_{s}+2 q\right] x_{(a} n_{b)},
\end{aligned}
$$

By contracting (82) in turn with the tensors $u^{a} u^{b}, x^{a} x^{b}, h^{a b}, u^{a} x^{b}, u^{a} h^{b c}$ and $x^{a} h^{b c}$ following equations are derived:

$$
\begin{gathered}
£_{\xi} \rho_{s}+2 \psi \rho_{s}=-2\left(\square \psi+\psi_{; a b} u^{a} u^{b}\right) \\
£_{\xi} \rho_{s}+2 \psi \rho_{s}=2\left(\square \psi-\psi_{; a b} x^{a} x^{b}\right) \\
£_{\xi} q+2 \psi q=2 \square \psi-\psi_{; a b} h^{a b}
\end{gathered}
$$




$$
\begin{gathered}
2 \psi_{; a b} u^{a} x^{b}=0 \\
\left(\rho_{s}+q\right) h^{b c} v_{b}=\psi_{; a b} u^{a} h^{b c} \\
\left(\rho_{s}+q\right) h^{b c} n_{b}=\psi_{; a b} x^{a} h^{b c}
\end{gathered}
$$

Equations (82)-(87) are valid for any $\mathrm{CKV} \xi^{a}$.

From Eq. (85) we have $\psi_{; a b}=0$, i.e., $\xi^{a}$ is always a SCKV in contrast to the cloud of strings. If $\xi^{a}$ is a SCKV or $\mathrm{HV}$ or $\mathrm{KV}$, then, there exists no curvature inheritance vector (CIV) other than a curvature collineation vector $(\mathrm{CCV})^{8}$. Therefore, string fluid doesn't admit CIV.

\section{Conclusions}

In the case of string cloud, we have found Eqs. (50), (51), (58) and (63) as inheritance equations. We have shown that the derivations of (58) and (63) required the reasonable assumptions contained in (55) and (59), i.e., $\rho_{p} \neq$ $0, \rho \neq 0$ and $\lambda \neq 0$.

We have also found under the conditions which $\xi^{a}$ is parallel to $x^{a}$ that there was a relation between $\rho$ and $\lambda$ as the equation of state $(\rho=\lambda)$ for a

cloud of geometric strings. Furthermore, we have found that $\rho=(1+w) \lambda$ which coincide with Takabayashi string when $\xi^{a}$ orthogonal to $x^{a}$ and $u^{a}$. For $\alpha=0$ and $\psi \neq 0$, we have equation of state $(\rho=\lambda)$ from Eqs. (75) and (77).

In the case of string fluids, we have found that $\xi^{a}$ is always a SCKV. So, we conclude that string doesn't admit CIV, i.e., string fluid admits CC (A $\mathrm{CC}$ is also a CKV iff $\psi_{; a b}=0$, see Ref. 7). If $\xi^{a}$ is a SCKV, then we have that

$$
\begin{gathered}
£_{\xi} q+2 \psi q=£_{\xi} \rho_{s}+2 \psi \rho_{s}=0, \\
£_{\xi} u_{a}=\psi u_{a} \text { and } £_{\xi} x_{a}=\psi x_{a}
\end{gathered}
$$

which is given by Yavuz and Yllmaz ${ }^{6}$ as inheritance equations. 


\section{Acknowledgment}

The authors would like to thank K. L. Duggal and J. Carot for providing us with preprints of their papers.

\section{References}

[1] K. YAno, Integral Formulas in Riemannian Geometry, Marcel Dekker, New York, 1970.

[2] L. Herrera and J. De Leon Ponce, J. Math. Phys. 26, 778, 2018, 2847 (1985).

[3] D. P. Mason and M. Tsamparlis, J. Math. Phys. 26, 2881 (1985).

[4] R. Maartens, D.P. Mason and M. Tsamparlis, J. Math. Phys. 27, 2987 (1986).

[5] A. A. Coley and B. O. J. Tupper, J. Math. Phys. 30, 2616 (1989).

[6] İ. Yavuz and İ. Yilmaz, Gen. Rel. Grav. 29, 1295 (1997).

[7] J. Carot, A. A. Coley And A. Sintes, Gen. Rel. Grav. 28, 311 (1996).

[8] K. L. Duggal, J. Math. Phys. 33, 2989 (1992).

[9] K. L. Duggal, Acta Applicandae Mathematicae, 31, 225 (1993).

[10] C. B. Kilinẹ And İ. Yavuz, Astrophys. Space Sci. 238, 239 (1996).

[11] İ. Yavuz and İ. Tarhan, Astrophys. Space Sci. 240, 45 (1996).

[12] İ. Yavuz and İ. Yilmaz, Astrophys. Space Sci. 245, 131 (1996).

[13] R. Bali And D. R. Jain, Astrophys. Space Sci. 141, 207 (1988).

[14] R. Bali and A. Tyagi, Int. J. Theor. Phys. 27, 627 (1988).

[15] R. BALI, Astrophys. Space Sci. 116, 81 (1985). 
[16] T. Singh and R. S. SRivastava, Astrophys. Space Sci. 159, 21 (1989).

[17] R. Bali and R. J. Deepak, Int. J. Theor. Phys. 28, 903 (1989).

[18] C. B. Kilinç, Astrophys. Space Sci. 222, 171 (1994).

[19] P. S. Letelier, Phys. Rev. D28, 2414 (1983).

[20] P. S. Letelier, Nuovo Cim.. B63, 519 (1981).

[21] P. S. Letelier, Phys. Rev.. D22, 807 (1980). 